\title{
Clinical characteristics of spontaneous coronary artery dissection in young female patients with acute myocardial infarction in Korea
}

Yongcheol Kim, Xiongyi Han*, Youngkeun Ahn, Min Chul Kim, Doo Sun Sim, Young Joon Hong, Ju Han Kim, and Myung Ho Jeong

Department of Cardiology, Chonnam National University Hospital, Gwangju, Korea
Background/Aims: We aimed to evaluate the prevalence, characteristics, and clinical outcomes of spontaneous coronary artery dissection (SCAD) in young female patients with acute myocardial infarction (AMI).

Methods: We identified 8,250 patients diagnosed with AMI who underwent coronary angiogram from the Chonnam National University Hospital database, Gwangju, Korea, between November 2005 and September 2017. A total of $148 \mathrm{fe-}$ male patients aged less than 60 years with a history of AMI were retrospectively studied and the characteristics and clinical outcomes were evaluated for all SCAD patients.

Results: Among female patients with AMI aged less than 60 years, the prevalence of SCAD was $8.78 \%$ (13 of 148). Based on the angiographic classification, type 2 SCAD was most commonly observed on angiograms in $69.2 \%$ of the cases (nine of 13), followed by type 3 in $23.1 \%$ (three of 13), and type 1 in $7.7 \%$ (one of 13). Furthermore, the left anterior descending (LAD) artery was the most commonly affected coronary artery $(76.9 \%, 10$ of 13 cases) and the distal segments of the coronary arteries were the most common sites of SCAD (92.3\%, 12 of 13). Regarding the clinical outcomes, one of 13 patients experienced repeat revascularization during the following 31 months.

Conclusions: The prevalence of SCAD was $8.7 \%$, indicating that SCAD is not rare, among female patients aged less than 60 years with AMI in Korea. Type 2 SCAD was most commonly observed on angiogram. Moreover, the distal portion of the LAD was the segment most commonly affected by SCAD. The long-term clinical outcomes were favorable in patients surviving SCAD.

Keywords: Dissection; Coronary artery disease; Myocardial infarction; Prevalence; Prognosis

\section{INTRODUCTION}

Although the true prevalence of spontaneous coronary artery dissection (SCAD) is still uncertain, SCAD has been considered to be an infrequent cause of acute coronary syndrome (ACS), including acute myocardial infarction (AMI), with a prevalence of approximately $0.16 \%$ to $2.0 \%$, detected on a coronary angiogram (CAG) [1-5]. However, the use of optical coherence tomography, providing a high resolution of $10 \mu \mathrm{m}$, enables detection intramural 

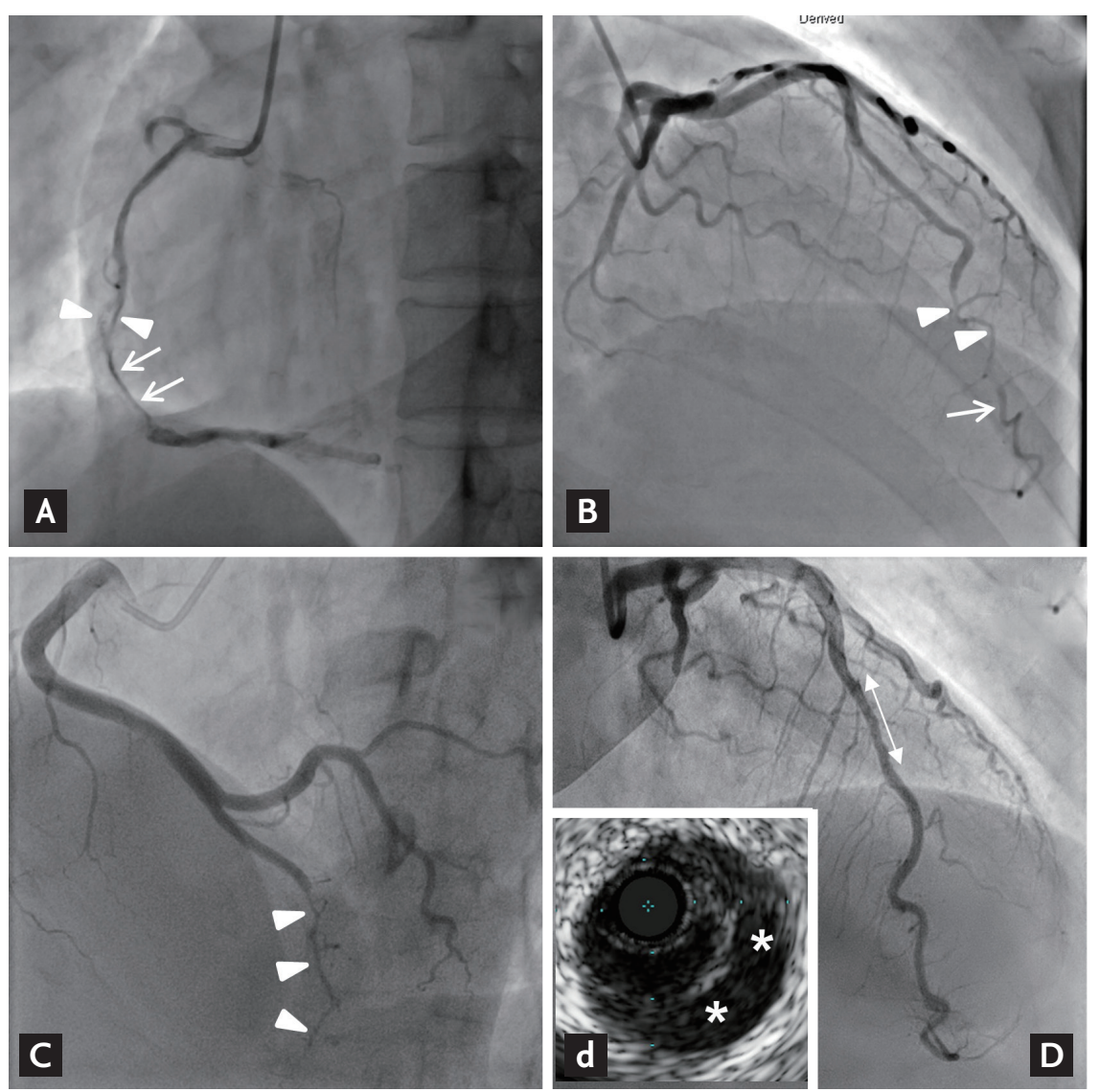

Figure 1. Angiographic classification of spontaneous coronary artery dissection. (A) Patient no. 9: type I, arterial wall contrast staining (arrows) or multiple radiolucent lumens (arrowheads). (B) Patient no. 7: type 2a, abrupt caliber reduction (arrowheads) with restoration of distal vessel caliber (arrow). (C) Patient no. 13: type $2 \mathrm{~b}$, abrupt caliber reduction without restoration of distal vessel caliber (arrowheads). (D) Patient no. 5: type 3, tubular stenosis mimicking atherosclerosis (double-point arrow), and confirming false lumen (asterisks) by intravascular ultrasound (d). hematoma and a false lumen for SCAD diagnosis, and it revealed a higher prevalence of $4.0 \%$ in ACS patients [6-9]. Furthermore, SCAD is a frequent cause of ACS in young female patients $[2-4,10]$.

With respect to the clinical outcomes, mid- to longterm mortality rate is low in patients who survive SCAD [3,11-13]. In contrast, the overall rate of major adverse cardiac events (MACE), such as death, recurrent AMI and urgent revascularization, is significant and it has been reported in $14.6 \%$ to $47.4 \%$ of cases during a 3 - to 10 -year follow-up period [11-13].

However, the data on SCAD in patients with AMI, especially young females who rarely have traditional cardiovascular risk factors, are limited, although AMI is the leading cause of mortality in the Asia-Pacific population, including Korea [14,15]. Therefore, the aim of this retrospective observational study was to investigate the prevalence, characteristics, and clinical outcomes of $\mathrm{SCAD}$ in young female patients with AMI in Korea.

\section{METHODS}

\section{Study population}

The study population was derived from the Chonnam National University Hospital database, Gwangju, Korea, between November 2005 and September 2017. Female patients aged less than 60 years with AMI were divided into two groups, namely, the SCAD and non-SCAD groups. We compared the baseline clinical characteristics, laboratory findings, and medication between the groups. Moreover, the clinical characteristics and outcomes were evaluated in all SCAD patients. The study protocol was approved by the Institutional Review Board of the Chonnam National University Hospital (approval number: CNUH 05-49) and the need for informed consent was waived.

\section{Angiographic classification of spontaneous coronary artery dissection \\ SCAD was diagnosed and classified according to the cri-}




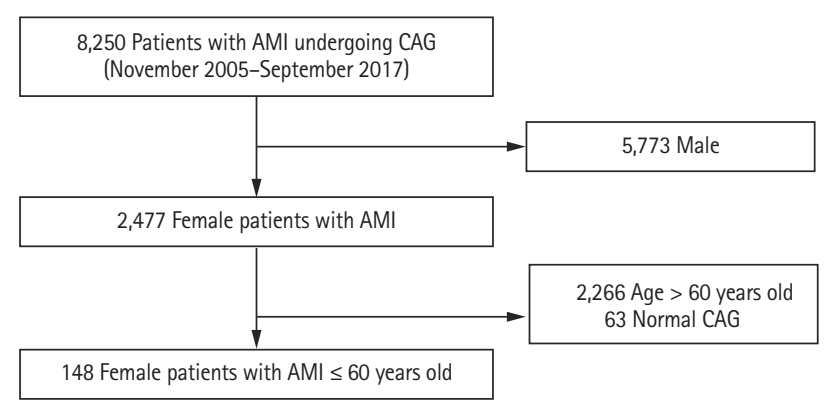

Figure 2. Flow chart of the study patients. AMI, acute myocardial infarction; CAG, coronary angiogram.

teria suggested by Saw as shown in Fig. 1 [16]. All CAGs were assessed for the diagnosis of SCAD by two independent interventional cardiologists (Y.K. and X.H.), who were blinded to the history of the patients. Type 1 refers to the classic appearance of arterial wall contrast staining or multiple radiolucent lumens (Fig. 1A). Type 2 refers to an abrupt caliber reduction from the normal diameter to diffuse smooth narrowing with or without the restoration of distal vessel caliber (type $2 \mathrm{a}$ and $2 \mathrm{~b}$ SCAD, respectively) (Fig. $1 B$ and ${ }_{1}$ C). Type 3 SCAD refers to tubular stenosis that mimics atherosclerosis requiring intracoronary imaging to confirm diagnosis (Fig. 1D). Regarding coronary artery segment classification, two interventional cardiologists determined each coronary artery segment according to the American Heart Association classification [17].

\section{Statistical analysis}

All continuous variables were expressed as mean with standard deviation (SD) or median with interquartile ranges (IQR), as appropriate. All categorical variables were reported as numbers with percentages. The continuous variables were compared using the unpaired $t$ test or Mann-Whitney $U$ test, as appropriate. The categorical variables were analyzed by chi-square test or Fisher's exact test. All analyses were two-tailed, and a $p$ $<0.05$ was considered significant. All statistical analyses were performed using SPSS for Windows software version 21.0 (IBM Co., Armonk, NY, USA).

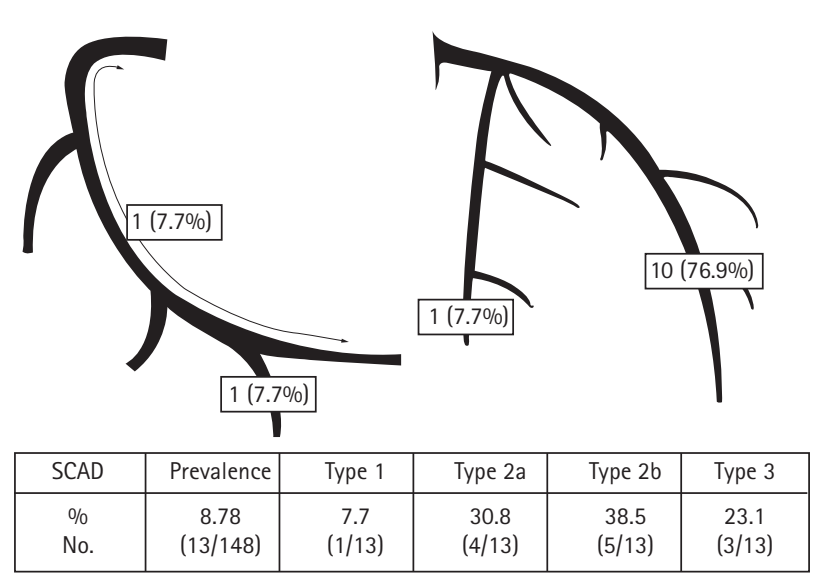

Figure 3. Prevalence, type, and location of spontaneous coronary artery dissection. SCAD, spontaneous coronary artery dissection.

\section{RESULTS}

Between November 2005 and September 2017, we identified 8,250 patients diagnosed with AMI who underwent CAG. Male $(\mathrm{n}=5,773)$ and female patients aged more than 60 years $(n=2,266)$ were excluded. Furthermore, patients with normal coronary artery $(n=63)$, defined as less than $30 \%$ stenosis on CAG by quantitative coronary angiography, were also excluded. Therefore, we selected a total of 148 female AMI patients aged less than 60 years (Fig. 2).

\section{Prevalence of SCAD in the study population}

From among 148 female patients with AMI aged less than 60 years, 13 patients presenting with SCAD were identified. Therefore, the overall prevalence of SCAD in this study population was $8.78 \%$ (13/148) (Fig. 3).

\section{Baseline characteristics and laboratory findings}

Baseline characteristics and laboratory findings are shown in Table 1. Mean age was similar for the SCAD and non-SCAD group ( $52.1 \pm 5.8$ vs. $53.7 \pm 5.7, p=0.350)$. There were no pregnant patients in either of the groups. With respect to the baseline characteristics, there was no significant difference between the two groups. As far as laboratory findings are concerned, lipid profiles were significantly different in the two groups. The salient difference here was that the high density lipoprotein cholesterol level was significantly higher in the SCAD group 
Table 1. Baseline and laboratory characteristics in the both SCAD and non-SCAD groups

\begin{tabular}{|c|c|c|c|}
\hline Variable & $\operatorname{SCAD}(\mathrm{n}=13)$ & Non-SCAD $(\mathrm{n}=135)$ & $p$ value \\
\hline \multicolumn{4}{|l|}{ Demographic } \\
\hline Age, yr & $52.1 \pm 5.8$ & $53 \cdot 7 \pm 5 \cdot 7$ & 0.350 \\
\hline $\mathrm{BMI}, \mathrm{kg} / \mathrm{m}^{2}$ & $21.4 \pm 8.2$ & $22.5 \pm 6.6$ & 0.596 \\
\hline Pregnancy & o & 0 & \\
\hline \multicolumn{4}{|l|}{ Vital sign on admission } \\
\hline $\mathrm{SBP}, \mathrm{mmHg}$ & $130.8 \pm 19.3$ & $124.7 \pm 31.9$ & 0.500 \\
\hline $\mathrm{DBP}, \mathrm{mmHg}$ & $82.3 \pm 10.9$ & $77 \cdot 3 \pm 21.3$ & 0.404 \\
\hline HR, beat/min & $80.6 \pm 19.1$ & $79.7 \pm 20.5$ & 0.882 \\
\hline \multicolumn{4}{|l|}{ Cardiovascular risk factors } \\
\hline Hypertension & $3(23.1)$ & $70(48.9)$ & 0.075 \\
\hline Diabetes mellitus & $1(7 \cdot 7)$ & $45(27 \cdot 4)$ & 0.120 \\
\hline Dyslipidemia & $1(7.7)$ & $13(8.9)$ & 0.884 \\
\hline Current smoking & $3(23.1)$ & $16(11.9)$ & 0.248 \\
\hline Menopause & $3(23.1)$ & $24(15 \cdot 6)$ & 0.482 \\
\hline \multicolumn{4}{|l|}{ Clinical manifestations } \\
\hline Chest pain & $12(92.3)$ & $109(80.7)$ & 0.302 \\
\hline Dyspnea & $1(7 \cdot 7)$ & $26(19 \cdot 3)$ & 0.302 \\
\hline Heart failure & o & $17(12.6)$ & 0.174 \\
\hline STEMI & $7(53.8)$ & $77(57.0)$ & 0.824 \\
\hline Killip classification III/IV & o & $21(15 \cdot 6)$ & 0.125 \\
\hline LVEF, \% & $54.9 \pm 8.9$ & $55.7 \pm 11.4$ & 0.818 \\
\hline \multicolumn{4}{|l|}{ Laboratory findings } \\
\hline Total cholesterol, mg/dL & $168(147-197)$ & $173(149-216)$ & 0.485 \\
\hline Triglyceride, mg/dL & $78(39-109)$ & $96(79-140)$ & 0.051 \\
\hline HDL-C, mg/dL & $54(46-69)$ & $40(35-53)$ & $<0.001$ \\
\hline LDL-C, mg/dL & $99(67-127)$ & $108(82-144)$ & 0.106 \\
\hline hsCRP, mg/dL & $0.8(0.1-2.9)$ & $0.8(0.2-3.5)$ & 0.546 \\
\hline Peak CK-MB, ng/dL & $38.2(2.6-145.5)$ & $33.3(7.9-104.2)$ & 0.790 \\
\hline Peak troponin-I, ng/dL & $5.1(1.1-21.1)$ & $17.1(4.7-67.4)$ & $<0.001$ \\
\hline
\end{tabular}

Values are presented as mean $\pm \mathrm{SD}$, number (\%), or median (interquartile range).

SCAD, spontaneous coronary artery dissection; BMI, body mass index; SBP, systolic blood pressure; DBP, diastolic blood pressure; HR, heart rate; STEMI, ST-elevation myocardial infarction; LVEF, left ventricular ejection fraction; HDL-C, high density lipoprotein cholesterol; LDL-C, low density lipoprotein cholesterol; hsCRP, high-sensitivity C-reactive protein; CK, creatine kinase.

$(p<0.001)$. Furthermore, the peak troponin-I level was significantly lower in the SCAD group than in the nonSCAD group (5.1 ng/dL vs. 17.1 ng/dL, $p<0.001$ ).

\section{Coronary angiographic findings and medication at discharge}

Coronary angiographic findings and medication at discharge are shown in Table 2 . With respect to the in- farct-related artery, there was no significant difference between the two groups. However, SCAD was not observed in the left main coronary artery. Lesion severity and Thrombolysis In Myocardial Infarction (TIMI) grade flow were similar between the two groups. Regarding discharge medication, antiplatelet agents, including aspirin and $\mathrm{P}_{2} \mathrm{Y}_{12}$ receptor inhibitor as the dual antiplatelet therapy (DAPT) (46.2\% vs. $97.8 \%, p<0.001)$, 
Table 2. Characteristics of coronary angiography and discharge medication in the two groups

\begin{tabular}{|c|c|c|c|}
\hline Variable & $\operatorname{SCAD}(\mathrm{n}=13)$ & Non-SCAD $(\mathrm{n}=135)$ & $p$ value \\
\hline Infarct-related artery & & & 0.313 \\
\hline Left anterior descending & $10(76.9)$ & $68(50.4)$ & \\
\hline Left circumflex & $1(7.7)$ & $24(17.8)$ & \\
\hline Right coronary & $2(15 \cdot 4)$ & $36(26.7)$ & \\
\hline Left main & 0 & $7(5.2)$ & \\
\hline ACC/AHA B2/C lesion & $9(69.2)$ & $117(86.7)$ & 0.091 \\
\hline \multicolumn{4}{|l|}{ TIMI grade flow } \\
\hline Initial TIMI grade flow o/1 & $6(46.2)$ & $59(43.7)$ & 0.865 \\
\hline Final TIMI grade flow $2 / 3$ & $12(92.3)$ & $133(98.5)$ & 0.129 \\
\hline \multicolumn{4}{|l|}{ Medication at discharge } \\
\hline Dual antiplatelet therapy & $6(46.2)$ & $132(97.8)$ & $<0.001$ \\
\hline Aspirin & $7(53.8)$ & $134(99 \cdot 3)$ & $<0.001$ \\
\hline $\mathrm{P}_{2} \mathrm{Y}_{12}$ receptor inhibitor & $10(76.9)$ & $132(97.8)$ & $<0.001$ \\
\hline Clopidogrel & $7(53.8)$ & $104(77.0)$ & \\
\hline Ticagrelor & $3(23.1)$ & $32(23.7)$ & \\
\hline Prasugrel & 0 & $24(17.8)$ & \\
\hline Statin & $9(69.2)$ & $118(87 \cdot 4)$ & 0.073 \\
\hline ACEi/ARB & $10(76.9)$ & $113(83.7)$ & 0.533 \\
\hline$\beta$-Blocker & $9(69.2)$ & $111(82.2)$ & 0.253 \\
\hline Calcium channel blocker & $4(30.8)$ & $18(13.8)$ & 0.091 \\
\hline
\end{tabular}

Values are presented as number (\%).

SCAD, spontaneous coronary artery dissection; ACC, American College of Cardiology; AHA, American Heart Association; TIMI, Thrombolysis In Myocardial Infarction; ACEi, angiotensin-converting enzyme inhibitor; ARB, angiotensin II receptor blocker.

aspirin (53.9\% vs. 99.3\%, $p<0.001$ ), and $\mathrm{P}_{2} \mathrm{Y} 12$ receptor inhibitor (76.9\% vs. $97.8 \%, p<0.001$ ), were less frequently prescribed to the SCAD group compared to the nonSCAD group.

\section{Characteristics of patients with SCAD}

Characteristics of patients with SCAD are shown in Table 3, Fig. 3. Regarding angiographic classification of $\mathrm{SCAD}$, type 1 was observed in one (7.7\%), type 2 was observed in nine (69.2\%) (type $2 \mathrm{a}$ in four [30.8\%] and type $2 \mathrm{~b}$ in five [38.5\%]), and type 3 was observed in three patients (23.1\%) (Fig. 3). Among 13 patients with SCAD, the coronary artery involved was the left anterior descending (LAD) artery, the right coronary artery, and the left circumflex artery in 10 (76.9\%), two (15.4\%), and one (7.7\%), respectively. Twelve SCAD lesions (93.3\%) were observed only in the distal portions of the coronary arteries. TIMI grade flow was o/1 in six (40\%), and all these patients were treated with percutaneous coronary intervention (PCI) with stent implantation. Intravascular ultrasound guidance was performed in four (30.8\%). The final TIMI grade flow of $2 / 3$ was achieved in most patients (92.3\%). During a median follow-up of 935 days (range, 121 to 4,125), one patient required repeat PCI (Table 3).

\section{DISCUSSION}

In this study of female patients under the age of 60 years with AMI, we found that the prevalence of SCAD was $8.78 \%$. Using angiographic classification, the most common SCAD angiographic appearance was type 2 in $69.2 \%$ of cases (nine of 13 ), followed by type 3 in $23.1 \%$ (three of 13), and type 1 in $7.7 \%$ (one of 13). Furthermore, SCAD was frequently observed in the $\operatorname{LAD}(76.9 \%$, 10 of 13) and the distal segments of the coronary arteries were 
Table 3. Characteristics of acute myocardial infarction patients with spontaneous coronary artery dissection aged less than 60 years

\begin{tabular}{|c|c|c|c|c|c|c|c|c|c|c|c|}
\hline No. & $\begin{array}{l}\text { Age, } \\
\text { yr }\end{array}$ & Diagnosis & $\begin{array}{l}\text { SCAD } \\
\text { type }\end{array}$ & Vessel & Location & $\begin{array}{c}\text { Initial } \\
\text { TIMI } \\
\text { grade flow }\end{array}$ & $\begin{array}{l}\text { Treat- } \\
\text { ment }\end{array}$ & $\begin{array}{l}\text { Intravascu- } \\
\text { lar imaging }\end{array}$ & $\begin{array}{c}\text { Final } \\
\text { TIMI } \\
\text { grade flow }\end{array}$ & $\begin{array}{c}\text { Follow-up, } \\
\text { day }\end{array}$ & $\begin{array}{c}\text { Outcomes } \\
\text { (event } \\
\text { day) }\end{array}$ \\
\hline 1 & 56 & NSTEMI & $2 a$ & LAD & Distal & 2 & $\begin{array}{l}\text { POBA } \\
\text { (failed) }\end{array}$ & No & 1 & 702 & No \\
\hline 2 & 54 & STEMI & $2 b$ & LAD & Distal & o & Stenting & No & 3 & 4,125 & $\begin{array}{l}\text { Repeat } \\
\text { PCI (236) }\end{array}$ \\
\hline 3 & 43 & STEMI & $2 b$ & LAD & Distal & o & Stenting & No & 3 & 121 & No \\
\hline 4 & 47 & NSTEMI & 3 & LAD & Distal & 2 & Medical & IVUS & 2 & 751 & No \\
\hline 5 & 54 & STEMI & 3 & LAD & Distal & 3 & Medical & IVUS & 3 & 733 & No \\
\hline 6 & 57 & NSTEMI & 3 & LAD & Distal & 2 & Medical & IVUS & 2 & 365 & No \\
\hline 7 & 43 & NSTEMI & $2 a$ & LAD & Distal & o & Stenting & No & 3 & 935 & No \\
\hline 8 & 58 & NSTEMI & $2 a$ & LCX & Distal & 2 & POBA & No & 3 & 1,399 & No \\
\hline 9 & 42 & STEMI & 1 & RCA & $\begin{array}{l}\text { Proximal- } \\
\text { distal }\end{array}$ & 1 & Stenting & IVUS & 3 & 2,438 & No \\
\hline 10 & 53 & NSTEMI & $2 a$ & LAD & Distal & 3 & Medical & No & 3 & 2,559 & No \\
\hline 11 & 54 & STEMI & $2 b$ & LAD & Distal & o & Stenting & No & 3 & 3,154 & No \\
\hline 12 & 54 & STEMI & $2 b$ & LAD & Distal & 0 & Stenting & No & 3 & 3,100 & No \\
\hline 13 & 45 & STEMI & $2 \mathrm{~b}$ & RCA & Distal & 3 & Medical & No & 3 & 325 & No \\
\hline
\end{tabular}

SCAD, spontaneous coronary artery dissection; TIMI, Thrombolysis In Myocardial Infarction; NSTEMI, non-ST-elevation myocardial infarction; LAD, left anterior descending artery; POBA, plain old balloon angioplasty; STEMI, ST-elevation myocardial infarction; PCI, percutaneous coronary intervention; IVUS, intravascular ultrasound; LCX, left circumflex artery; RCA, right coronary artery.

the most common sites of SCAD (92.3\%, 12 of 13). Regarding the clinical outcomes, one of 13 patients experienced repeat revascularization during the following 31 months. To our knowledge, this is the first Korea study reporting the prevalence and characteristics of SCAD patients presenting with AMI.

Several studies have assessed the prevalence of SCAD in young female patients with ACS including ST-elevation myocardial infarction (STEMI). Vanzetto et al. [2] reported that the prevalence of SCAD was $8.7 \%$ (12 of 138) in female ACS patients under 50 years of age and reached $10.8 \%$ (eight of 74 ) in cases of STEMI. However, the prevalence was reported as $24.2 \%$ (16 of 66) among young women with AMI (aged $\leq 50$ ) in a retrospective single-center study [10]. Another retrospective single-center study reported an identical prevalence of $24.2 \%$ ( 16 of 66 ) in female patients under the age of 60 years with AMI [4]. Moreover, the Japanese multicenter registry described a prevalence of SCAD of $34.6 \%$ ( 45 of 130) in female patients aged under 50 with AMI [3]. Although it is difficult to explain the discrepancy in the prevalence of SCAD in these different studies, the present study supported the view that SCAD is not rare among young female AMI patients undergoing CAG. Therefore, interventional cardiologists must consider SCAD in the differential diagnosis of the etiology of AMI in young women.

Several studies have reported that the most common angiographic appearance is type 2 in $52 \%$ to $67 \%$ of all SCAD cases, which is comparable to the results in the present study $[3,18,19]$. With respect to the affected artery and segment, the LAD and mid to distal segments are each commonly affected [18]. The present study also showed similar results in that the involvement of the LAD and distal segments is common, albeit in a relatively higher proportion than in other studies. Therefore, interventional cardiologists should look carefully at the distal segment of the LAD when performing CAG in young female patients presenting with AMI.

Expert opinion supports the view that overall conservative treatment is preferable in patients with SCAD as spontaneous healing of the affected artery was observed 
in most cases with medical treatment and PCI was associated high failure rates $[16,20,21]$. However, Buccheri et al. [22] reported that physicians preferred invasive treatment in about $40 \%$ of SCAD patients presenting with ACS, although most of the SCAD patients were treated conservatively in the literature surveyed. In our study, the patients with TIMI grade flow o/1 were treated with PCI with stent implantation. Among patients with TIMI grade flow 2/3, two patients were treated with PCI with plain old balloon angioplasty, but PCI failure (TIMI grade flow 2 to 1 ) was observed in one case (patient no. 1). In contrast, the present study showed good long-term clinical outcomes in patients with TIMI grade flow $2 / 3$ who treated with medical treatment. Therefore, conservative treatment may be a good management option for SCAD patients presenting with AMI, unless there is flow limitation.

There is a scarcity of data regarding the role of DAPT in SCAD patients. Patients with SCAD who undergo stenting should receive DAPT according to current revascularization guidelines [23]. In the present study, DAPT was prescribed in all SCAD patients who were treated with stent implantation (six of six). With respect to SCAD patients with conservative management, a further prospective study should be needed to confirm the benefit of antiplatelet therapy.

With regard to the clinical outcomes, several studies have reported that long-term mortality is low, approximately $\% \%$ to $8.0 \%$ during a follow-up of 2.8 to 10 years $[3,11-13,18]$. This study showed that the prognosis was favorable and the frequency of cardiovascular events was low with only one patient (7.7\%) requiring repeat PCI due to a stent fracture. In this patient, subsequent follow-up of 10.8 years revealed no recurrence of cardiovascular events. Except for one adverse event, there were no MACE including death during the follow-up period.

There were several limitations in the present study. Firstly, this study was a single-center, retrospective study with the inherent limitations of a small sample size. Therefore, selection bias was virtually unavoidable. Secondly, in relation to patients with type 3 SCAD, we included only those who were confirmed by an intravascular imaging tool. This might have led to underestimation of the prevalence of type $3 \mathrm{SCAD}$ as patients in this category who did not undergo intravascular imaging might have been inadvertently excluded. Furthermore, there was no evaluation of lesion characteristics with intravascular imaging to confirm a diagnosis in patients with type 2 SCAD. Therefore, the prevalence of type 2 SCAD might have been overestimated in this study. Thirdly, we did not examine the possibility of fibromuscular dysplasia, the most frequent predisposing arteriopathy, in the SCAD group. Despite these limitations, this study is expected to prove helpful to physicians in understanding the characteristics of patients with SCAD in Korea.

In conclusion, prevalence of SCAD was $8.78 \%$ in female patients with AMI under the age of 60 years. Moreover, type 2 and involvement of the distal portion of the LAD were the most common angiographic appearance and segment of SCAD, respectively. Long-term clinical outcomes were favorable in patients surviving SCAD. Therefore, increased awareness of SCAD is needed when CAG is performed in young female patients presenting with AMI as SCAD is not rare in these patients.

\section{KEY MESSAGE}

1. Between 2005 and 2017 , the prevalence of spontaneous coronary artery dissection (SCAD) was $8.78 \%$ among female patients aged less than 60 years with acute myocardial infarction from single-center data in Korea.

2. Type 2 SCAD was most commonly observed on angiograms, and the distal portion of the left anterior descending artery was the most commonly affected segment in the patients.

3. Long-term clinical outcomes were favorable in patients surviving SCAD.

\section{Conflict of interest}

No potential conflict of interest relevant to this article was reported.

\section{Acknowledgments}

This study was supported by a grant from Chonnam National University Hospital Biomedical Research Institute (BCRI8015) and by a grant from the Korean Health Technology R\&D Project, Ministry of Health and Welfare, Republic of Korea ( $\left.\mathrm{HI}_{13} \mathrm{C} 1527\right)$. 


\section{REFERENCES}

1. Alfonso F, Bastante T. Spontaneous coronary artery dissection: novel diagnostic insights from large series of patients. Circ Cardiovasc Interv 2014;7:638-641.

2. Vanzetto G, Berger-Coz E, Barone-Rochette G, et al. Prevalence, therapeutic management and medium-term prognosis of spontaneous coronary artery dissection: results from a database of 11,605 patients. Eur J Cardiothorac Surg 2009;35:250-254.

3. Nakashima T, Noguchi T, Haruta S, et al. Prognostic impact of spontaneous coronary artery dissection in young female patients with acute myocardial infarction: a report from the Angina Pectoris-Myocardial Infarction Multicenter Investigators in Japan. Int J Cardiol 2016;207:341-348.

4. Rashid HN, Wong DT, Wijesekera $\mathrm{H}$, et al. Incidence and characterisation of spontaneous coronary artery dissection as a cause of acute coronary syndrome: a single-centre Australian experience. Int J Cardiol 2016;202:336-338.

5. Mortensen KH, Thuesen L, Kristensen IB, Christiansen EH. Spontaneous coronary artery dissection: a Western Denmark Heart Registry study. Catheter Cardiovasc Interv 2009;74:710-717.

6. Kim Y, Johnson TW, Akasaka T, Jeong MH. The role of optical coherence tomography in the setting of acute myocardial infarction. J Cardiol 2018;72:186-192.

7. Kim Y, Deharo P, Adlam D, Baumbach A, Johnson TW. The role of optical coherence tomography in decision making during the acute phase of spontaneous coronary artery dissection. Int J Cardiol Heart Vasc 2016;14:6-7.

8. Kim Y, Jeong MH, Kim MC, et al. Assessment for ambiguous angiographic finding in patient with acute myocardial infarction by optical coherence tomography. Cardiol J 2018;25:536-537.

9. Nishiguchi T, Tanaka A, Ozaki Y, et al. Prevalence of spontaneous coronary artery dissection in patients with acute coronary syndrome. Eur Heart J Acute Cardiovasc Care 2016;5:263-270.

10. Saw J, Aymong E, Mancini GB, Sedlak T, Starovoytov A, Ricci D. Nonatherosclerotic coronary artery disease in young women. Can J Cardiol 2014;30:814-819.

11. Tweet MS, Hayes SN, Pitta SR, et al. Clinical features, management, and prognosis of spontaneous coronary artery dissection. Circulation 2012;126:579-588.

12. Lettieri C, Zavalloni D, Rossini R, et al. Management and long-term prognosis of spontaneous coronary artery dissection. Am J Cardiol 2015;116:66-73.

13. Saw J, Humphries K, Aymong E, et al. Spontaneous coronary artery dissection: clinical outcomes and risk of recurrence. J Am Coll Cardiol 2017;70:1148-1158.

14. Kim Y, Jeong MH, Ahn Y, et al. Results of a 10-year experience in Korea using drug-eluting stents during percutaneous coronary intervention for acute myocardial infarction (from the Korea Acute Myocardial Infarction Registry). Am J Cardiol 2018;122:365-373.

15. Kim Y, Ahn Y, Cho MC, Kim CJ, Kim YJ, Jeong MH. Current status of acute myocardial infarction in Korea. Korean J Intern Med 2019;34:1-10.

16. Saw J, Mancini GBJ, Humphries KH. Contemporary review on spontaneous coronary artery dissection. J Am Coll Cardiol 2016;68:297-312.

17. Austen WG, Edwards JE, Frye RL, et al. A reporting system on patients evaluated for coronary artery disease. Report of the Ad Hoc Committee for Grading of Coronary Artery Disease, Council on Cardiovascular Surgery, American Heart Association. Circulation 1975:51(4 Suppl):5-40.

18. Saw J, Aymong E, Sedlak T, et al. Spontaneous coronary artery dissection: association with predisposing arteriopathies and precipitating stressors and cardiovascular outcomes. Circ Cardiovasc Interv 2014;7:645-655.

19. Rogowski S, Maeder MT, Weilenmann D, et al. Spontaneous coronary artery dissection: angiographic follow-up and long-term clinical outcome in a predominantly medically treated population. Catheter Cardiovasc Interv 2017;89:59-68.

20. Adlam D, Alfonso F, Maas A, Vrints C; Writing Committee. European Society of Cardiology, Acute Cardiovascular Care Association, SCAD study group: a position paper on spontaneous coronary artery dissection. Eur Heart J 2018;39:3353-3368.

21. Hayes SN, Kim ESH, Saw J, et al. Spontaneous coronary artery dissection: current state of the science: a scientific statement from the American Heart Association. Circulation 2018;137:e523-e557.

22. Buccheri D, Zambelli G, Alfonso F, Cortese B. Pulse on spontaneous coronary artery dissections: experience-based survey. JACC Cardiovasc Interv 2017;10:1469-1471.

23. Neumann FJ, Sousa-Uva M, Ahlsson A, et al. 2018 ESC/ EACTS guidelines on myocardial revascularization. Eur Heart J 2019;40:87-165. 